\title{
THE DOMINION AND SEPARABLE SUBALGEBRAS OF FINITELY GENERATED ALGEBRAS 1
}

\author{
DEAN SANDERS
}

ABSTRACT. Let $R \subseteq S \subseteq A$ be rings with $R$ commutative, $A$ an $R$-algebra which is finitely generated as an $R$-module by $n$-elements, and $S$ a subalgebra of $A$. Setting $D_{0}=S$ and $D_{i}=\operatorname{Dom}\left(R, D_{i-1}\right)$ for $i \geq 1$, we show that $D_{n}=R$. We use this to show that if $S$ is a separable $R$-algebra then $S$ is also finitely generated as an $R$-module. Finally, a characterization of $S$ is given when $A$ is commutative and $S$ is an inertial subalgebra of $A$.

1. Preliminaries. Throughout this paper all rings have identity, subrings contain the identity of the larger ring, and ring homomorphisms map the identity to the identity. With the exception of Definition 1 and Theorem $1, R$ denotes a commutative ring and $S$ denotes an $R$-algebra; that is, $S$ is a ring together with a ring homomorphism $f$ from $R$ into the center of $S$. It will be no real restriction to assume that $S$ is faithful as an $R$-module under the module structure induced by $f$; thus, we may consider $R$ as a subring of the center of $S$.

In any category a morphism $f$ is called an epimorphism if for any two morphisms $g$ and $h$ the equality of $g \circ f$ and $h \circ f$ implies the equality of $g$ and $h$. A ring $S$ will be called an epimorphic extension of a commutative ring $R$ if $S$ is a faithful $R$-algebra and the natural injection of $R$ into $S$ is an epimorphism.

2. The dominion of $R$ in $S$.

Definition 1. Let $R$ and $S$ be rings, not necessarily commutative, and let $f: R \rightarrow S$ be a ring homomorphism. The f-dominion of $R$ in $S$, denoted

Received by the editors June 21, 1973 and, in revised form, December 3, 1973. AMS (MOS) subject classifications (1970). Primary 16A16, 18A20.

Key words and phrases. Dominion, epimorphism, separable subalgebra, inertial subalgebra.

1This paper is based on a portion of the author's doctoral dissertation written at Michigan State University under the direction of Dr. E. C. Ingraham. 
$\operatorname{Dom}_{f}(R, S)$, is the set of elements $s$ of $S$ such that: If $g$ and $h$ are any two ring homomorphisms from $S$ to a ring $T$ with $g \circ f=h \circ f$, then $g(s)=$ $h(s)[5, \mathrm{p} .382]$.

It is easily seen that $\operatorname{Dom}_{f}(R, S)$ is a subring of $S$ containing $f(R)$. In addition $f: R \rightarrow S$ is an epimorphism if and only if $\operatorname{Dom}_{f}(R, S)=S$. Whenever $R$ is a subring of $S$ and $f$ is the natural injection of $R$ into $S$, we write $\operatorname{Dom}(R, S)$.

The following theorem gives two of the many characterizations of $\operatorname{Dom}_{f}(R, S)$.

Theorem 1. Let $R$ and $S$ be rings, not necessarily commutative, and let $f: R \rightarrow S$ be a ring homomorphism. The following statements are equiv. alent:

(1) $s \in \operatorname{Dom}_{f}(R, S)$.

(2) The relation $s \otimes 1-1 \otimes s=0$ holds in $S \otimes_{R} S$.

(3) There exist matrices $\sigma_{1}$ and $\sigma_{2}$ with entries from $S$ and a matrix $\theta$ with entries from $f(R)$ such that:

(i) $(s)=\sigma_{1} \theta \sigma_{2}$ and

(ii) both $\sigma_{1} \theta$ and $\theta \sigma_{2}$ have entries from $f(R)$.

Proof. The equivalence of (1) and (2) has been shown by Roby [4, Theorem 1]. The equivalence of (1) and (3) has been shown by Mazet [3, Theorem 1].

Due to the nature of the computations in the remainder of this section, some preliminaries are in order.

Let $S$ be a ring and $M$ a left $S$-module. Let $\mathcal{S}$ and $M$ denote the set of matrices having entries from $S$ and $M$ respectively. Define addition in $M$ and $\mathcal{S}$ and multiplication in $\mathcal{S}$ as usual. If $\sigma=\left(s_{i j}\right) \in \mathcal{S}$ is $m \times n$ and $\mu=$ $\left(m_{i j}\right) \in \mathbb{M}$ is $n \times p$ we define, quite naturally, $\sigma \mu$ to be the $m \times p$ matrix whose $i, j$ entry is $\sum_{k=1}^{n} s_{i k} m_{k j}$. If $\mu$ is $m \times n$ and $\sigma$ is $n \times p$ we define $\mu \cdot \sigma=\left(\sigma^{t} \mu^{t}\right)^{t}$ where ()$^{t}$ denotes the transpose matrix. If $x \in M$ we define $\sigma x$ to be the matrix $\left(s_{i j} x\right)$ (resp. $x \sigma=\left(x s_{i j}\right)$ whenever $M$ is a right $S$-module).

Suppose $\sigma, \sigma^{\prime}$ are in $\mathcal{S}, \mu, \mu^{\prime}$ are in $\mathbb{M}$, and $x$ is in $M$. Whenever these matrices have compatible dimensions they satisfy the following:

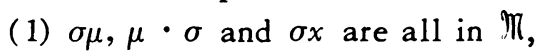

(2) $\left(\sigma+\sigma^{\prime}\right) \mu=\sigma \mu+\sigma^{\prime} \mu$,

(2') $\mu \cdot\left(\sigma+\sigma^{\prime}\right)=\mu \cdot \sigma+\mu \cdot \sigma^{\prime}$,

(3) $\sigma\left(\mu+\mu^{\prime}\right)=\sigma \mu+\sigma \mu^{\prime}$,

(3') $\left(\mu+\mu^{\prime}\right) \cdot \sigma=\mu \cdot \sigma+\mu^{\prime} \cdot \sigma$,

(4) $\left(\sigma \sigma^{\prime}\right) \mu=\sigma\left(\sigma^{\prime} \mu\right)$,

(5) $\left(\sigma \sigma^{\prime}\right) x=\sigma\left(\sigma^{\prime} x\right)$. 
If $S$ is commutative, then

(6) $\mu \cdot\left(\sigma \sigma^{\prime}\right)=(\mu \cdot \sigma) \cdot \sigma^{\prime}$,

(7) $\sigma\left(\sigma^{\prime} x\right)=(\sigma x) \cdot \sigma^{\prime}$.

Properties $2^{\prime}, 3^{\prime}$ and $1-5$ are immediate. Properties 6 and 7 follow from the identity $\left(\sigma \sigma^{\prime}\right)^{t}=\sigma^{t} \sigma^{t}$, which holds whenever $S$ is commutative.

We are now ready to proceed.

Proposition 2. Let $R$ be a commutative ring, $S$ a faithful $R$-algebra, and $M$ a two-sided S-module. If $m \in M$ is such that $r m=m r$ for every $r \in R$, then $s m=m s$ for every $s \in \operatorname{Dom}(R, S)$.

Proof. Suppose $m \in M$ is such that $r m=m r$ for every $r \in R$. If $s \in$ $\operatorname{Dom}(R, S)$ and if $\sigma_{1}, \sigma_{2}$ and $\theta$ are the matrices of Theorem 1 , then we have

$$
\begin{aligned}
(s) m & =\left(\sigma_{1} \theta \sigma_{2}\right) m=\sigma_{1}\left[\left(\theta \sigma_{2}\right) m\right]=\sigma_{1}\left[m\left(\theta \sigma_{2}\right)\right] \\
& =\sigma_{1}\left[(m \theta) \sigma_{2}\right]=\sigma_{1}\left[(\theta m) \sigma_{2}\right]=\left[\left(\sigma_{1} \theta\right) m\right] \sigma_{2} \\
& =\left[m\left(\sigma_{1} \theta\right)\right] \sigma_{2}=m\left(\sigma_{1} \theta \sigma_{2}\right)=m(s) .
\end{aligned}
$$

Therefore $s m=m s$.

Corollary 2.1. If $R$ is commutative and $S$ is a faithful $R$-algebra, then $\operatorname{Dom}(R, S)$ is contained in the center of $S$.

Proof. Set $M=S$ and note that $R$ is in the center of $S$.

Corollary 2.2. Any epimorphic extension of a commutative ring is itself commutative.

Next we will examine finitely generated $S$-modules.

Lemma 3. Let $S$ be an R-algebra and let $M$ be a left $S$-module. If a left $R$-submodule $N$ of $M$ and an element $x$ of $M$ are such that $M=N+R x$, then $N$ is a left $\operatorname{Dom}(R, S)$-submodule of $M$.

Proof. Let $s$ be in $\operatorname{Dom}(R, S)$ and let $n$ be in $N$; it suffices to show that $s n \in N$. Let $\sigma_{1}, \sigma_{2}$ and $\theta$ be the matrices of Theorem 1. Since $M=$ $N+R x$ we can set $\sigma_{1} x=\mu_{1}+\phi_{1} x$ and $\sigma_{2} n=\mu_{2}+\phi_{2} x$, where $\mu_{1}$ and $\mu_{2}$ are matrices with entries from $N$ and $\phi_{1}$ and $\phi_{2}$ are matrices with entries from $R$.

Having established notation we proceed. 


$$
\begin{aligned}
(s n)=\sigma_{1} \theta\left(\sigma_{2} n\right) & =\left(\sigma_{1} \theta\right) \mu_{2}+\sigma_{1}\left[\left(\theta \phi_{2}\right) x\right] \\
& =\left(\sigma_{1} \theta\right) \mu_{2}+\left(\sigma_{1} x\right) \cdot\left(\theta \phi_{2}\right) \\
& =\left(\sigma_{1} \theta\right) \mu_{2}+\mu_{1} \cdot\left(\theta \phi_{2}\right)+\left(\phi_{1} x\right) \cdot\left(\theta \phi_{2}\right) \\
& =\left(\sigma_{1} \theta\right) \mu_{2}+\mu_{1} \cdot\left(\theta \phi_{2}\right)+\phi_{1} \theta\left(\phi_{2} x\right) \\
& =\left(\sigma_{1} \theta\right) \mu_{2}+\mu_{1} \cdot\left(\theta \phi_{2}\right)+\phi_{1} \theta\left(\sigma_{2} n\right)-\left(\phi_{1} \theta\right) \mu_{2} \\
& =\left(\sigma_{1} \theta\right) \mu_{2}+\mu_{1} \cdot\left(\theta \phi_{2}\right)+\phi_{1}\left(\theta \sigma_{2}\right) n-\left(\phi_{1} \theta\right) \mu_{2} \cdot
\end{aligned}
$$

Since $N$ is a left $R$-module, the last equality expresses ( $s n$ ) as a sum of four $1 \times 1$ matrices each having its entry from $N$. Therefore $s n \in N$.

Theorem 4. Let $S$ be an R-algebra and let $M$ be a left $S$-module which is finitely generated as a left $R$-module by $n$ elements. Set $D_{0}=S$ and $D_{i}=\operatorname{Dom}\left(R, D_{i-1}\right), i \geq 1$. Then for any element $x$ of $M, D_{n} x=R x$.

Proof. Suppose $M$ is generated as a left $R$-module by $\left\{x_{1}, \ldots, x_{n}\right\}$. Set $x=x_{0}$ and $M_{i}=\sum_{j=0}^{n-i} R x_{j}, 0 \leq i \leq n$. Using Lemma 3 and a straightforward induction on $i$, we see that $M_{i}$ is a left $D_{i}$-module for each $i$. In particular, $M_{n}=R x$ is a left $D_{n}$-module. Therefore $D_{n} x \subseteq M_{n}=R x$ since $x \in R x$. Since $R \subseteq D_{i}$ for every $i$, it follows that $R x \subseteq D_{n} x$ and we are done.

Corollary 4.1. Let $R \subseteq S \subseteq A$ be rings with $R$-commutative, $A$ an $R$-algebra which is finitely generated as an $R$-module by $n$ elements, and $S$ a subalgebra of $A$. Then $D_{n}=R$.

Proof. Set $M=A$ and $x=1$ in Theorem 4.

The next corollary is a strengthening of the well-known result that if an epimorphic extension $S$ of $R$ is finitely generated as an $R$-module, then $S=R[4$, Theorem 2].

Corollary 4.2. Let $S$ be an epimorphic extension of $R$ and let $A$ be a ring containing $S$. If $A$ is finitely generated as a left $R$-module, then $S=$ $R$.

Proof. Let $M=A$ and $x=1$ in Theorem 4 and note that $D_{i}=S$ for every $i$.

3. Separable algebras. An $R$-algebra $S$ is $R$-separable if and only if $s \odot_{R} s^{0}$ contains an idempotent $e$ such that $(s \otimes 1-1 \otimes s) e=0$ for every $s$ in $S$ and $\mu(e)=1$, where $\mu$ is the multiplication map from $S \odot_{R} S^{0}$ to $S$ 
defined by $\mu\left(\Sigma s_{i} \otimes s_{i}^{\prime}\right)=\Sigma s_{i} s_{i}^{\prime}$. The idempotent $e$ is called a separability idempotent for $S$ over $R$ and is unique whenever $S$ is commutative [1, p. 40].

Life would be easier if separable algebras were finitely generated as modules; however, such is not the case. For example, let $R$ be a commutative ring and let $S$ be any (faithful) ring of quotients of $R$. For each element $r / w$ of $S$ one has the following relations in $S \otimes_{R} S$ :

$$
\frac{r}{w} \otimes 1=\frac{r}{w} \otimes w\left(\frac{1}{w}\right)=\frac{r w}{w} \otimes \frac{1}{w}=\frac{r}{1} \otimes \frac{1}{w}=1 \otimes \frac{r}{w}
$$

Therefore, $S$ is an epimorphic, a fortiori separable, $R$-algebra. However, $S$ cannot be finitely generated as an $R$-module unless $S=R$. The next theorem, which is the main result of this paper, offers a condition under which a separable algebra is finitely generated. The proof makes use of the following results:

If $S$ is a separable $R$-algebra with center $C$, then $S$ is finitely generated and projective as a $C$-module [1, Theorem 3.4]. In addition, $S$ is separable as a $C$-algebra and $C$ is separable as an $R$-algebra [1, Theorem 3.8].

We are now ready for the main result of this paper.

Theorem 5. Let $R$ be a commutative ring and let $A$ be an $R$-algebra which is finitely generated as an $R$-module. Then any $R$-separable subalgebra of $A$ is also finitely generated as an $R$-module.

Proof. Let $S$ be an $R$-separable subalgebra of $A$ and let $C$ denote the center of $S$. Since $S$ is finitely generated as a $C$-module, it suffices to prove that $C$ is finitely generated as an $R$-module. Since $C$ is commutative and is separable as an $R$-algebra, it has a unique separability idempotent $e=$ $\sum_{i=1}^{n} x_{i} \otimes y_{i}$ in $C \otimes_{R} C$. Let $R^{\prime}$ be the $R$-algebra generated by $\left\{x_{1}, \ldots, x_{n}\right\}$. Then $R^{\prime}$ is a subring of $C$. Since $A$ is finitely generated as an $R$-module, each element of $A$ is integral over $R$; therefore, $R^{\prime}$ is finitely generated as an $R$-module [6, pp. 254-255]. We will use Corollary 4.2 to show that $R^{\prime}=$ C.

Since $C$ is separable as an $R$-algebra, it is separable as an $R^{\prime}$-algebra. It is easy to see that $e^{\prime}=\sum_{i=1}^{n} x_{i} \otimes y_{i}$ in $C \otimes_{R^{\prime}} C$ is the unique separability idempotent for $C$ over $R^{\prime}$. However, each $x_{i}$ is in $R^{\prime}$ so that $e^{\prime}=1 \otimes \sum_{x=1}^{n} x_{i} y_{i}=1 \otimes \mu(e)=1 \otimes 1$ in $C \otimes_{R^{\prime}} C$. It is shown on p. 40 of [1] that $C \otimes_{R^{\prime}} C=\left(C \otimes_{R^{\prime}} C\right) e^{\prime} \otimes\left(C \otimes_{R^{\prime}} C\right)\left(1-e^{\prime}\right)$ where $\left(C \otimes_{R^{\prime}} C\right)\left(1-e^{\prime}\right)$ is the ideal in $C \otimes_{R}, C$ generated by elements of the form $s \otimes 1-1 \otimes s$. Since $1-e^{\prime}=0$ one concludes $s \otimes 1-1 \otimes s=0$ for all $s \in C$ so by Theorem 
1(2) $C$ is an epimorphic image of $R^{\prime}$ with $R^{\prime} \subseteq C \subseteq A$ and $A$ finitely generated as a left $R^{\prime}$-module. Applying Lemma 4.2 we have $C=R^{\prime}$ which is finitely generated as an $R$-module. Hence $S$ is finitely generated as an $R$-module.

We conclude this paper with a uniqueness theorem for inertial subalgebras.

Let $A$ be a finitely generated $R$-algebra with Jacobson radical $N$. A subalgebra $S$ of $A$ is called an inertial subalgebra if $S$ is a separable $R$ algebra such that $A=S+N[2$, Definition 2.1].

Lemma 6. Let $A$ be a finitely generated $R$-algebra with radical $N$. If $B$ and $S$ are subalgebras with $B \subseteq S, S$ separable over $R$, and $B+N=A$, then $B=S$.

Proof. Clearly $S$ is an inertial subalgebra of $A$ so that $\operatorname{rad} S=S \cap N$ [2, Lemma 1.1]. By Theorem $5, S$ is finitely generated as an $R$-module; thus, $\operatorname{rad} S=\bigcap(\mathrm{m} S)$ where $m$ runs over all maximal ideals of $R[2$, Lemma 1.1]. Therefore, $S=A \cap S=(B+N) \cap S=B+(N \cap S)=B+\bigcap(\mathrm{m} S)$, which implies $S=B+S$ for every maximal ideal $m$ of $R$. It follows from the generalized Nakayama lemma [6, Lemma 2, p. 215] that $S=B$.

Proposition 7. Let $A$ be a commutative, finitely generated $R$-algebra with radical $N$. If $S$ is an inertial subalgebra of $A$, then $S=\bigcap\{B \mid B$ is a subalgebra of $A$ with $A=B+N\}$.

Proof. Since $A=S+N$ the intersection is contained in $S$. Let $B$ be a subalgebra of $A$ such that $A=B+N$. The subalgebra $B S$ is a ring homomorphic image of $B \otimes{ }_{R} S$ and so is a separable $B$-algebra containing $B$. Applying Lemma 6 with $R=B$, we have $B=B S$ which contains $S$. Therefore, $S \subseteq \bigcap\{B \mid B$ is a subalgebra of $A$ with $A=B+N\}$.

Corollary 7.1. Let $A$ be a commutative, finitely generated R-algebra. An inertial subalgebra of $A$, if it exists, is unique.

\section{REFERENCES}

1. F. DeMeyer and E. C. Ingraham, Separable algebras over commutative rings, Lecture Notes in Math., vol. 181, Springer-Verlag, New York and Berlin, 1971. MR $43 \# 6199$.

2. E. C. Ingraham, Inertial subalgebras of algebras over commutative rings, Trans. Amer. Math. Soc. 124 (1966), 77-93. MR 34 \# 209. 
3. P. Mazet, Caractérisations des épimorphismes par relations et générateurs, Séminaire d'algèbre commutative dirigé par Pierre Samuel: 1967/68, Les épimorphismes d'anneaux, Secrétariat mathématique, Paris, 1968. MR 39 \#6867.

4. N. Roby, Diverses caractérisations des épimorphismes, Séminaire d'algèbre commutative dirigé par Pierre Samuel: 1967/68, Les épimorphismes d'anneaux, Secrétariat mathématique, Paris, 1968. MR 39 \#6867.

5. H. H. Storrer, Epimorphismen von kommutativen Ringen, Comment. Math. Helv. 43 (1968), 378-401. MR 39 \# 4137.

6. O. Zariski and P. Samuel, Commutative algebra. Vol. I, University Series in Higher Math., Van Nostrand, Princeton, N. J., 1958. MR 19, 833.

DEP ARTMENT OF MATHEMATICS, MICHIGAN STATE UNIVERSITY, EAST LANSING, MICHIGAN 48823

Current address: Department of Mathematics, Capital University, Columbus, Ohio 43209 